\title{
Construção de um sistema de monitoramento de grandezas elétricas aplicadas em um Motor de Indução bifásico.
}

\author{
Vitor Souza e Souza* Florindo Antônio de Carvalho Ayres Junior* Renan Landau Paiva de Medeiros* Hélder da Silva \\ Cruz* Luiz Miguel Andrade Conceição*. Nei Junior da Silva Farias*. Rafael da Silva Mendonça*. Mario Ruben Lima \\ de Oliveira**
}

\begin{abstract}
*Faculdade de Tecnologia, Universidade Federal do Amazonas, AM (e-mails: vitor.souza951@gmail.com, florindoayres@ufam.edu.br, renanlandau@ufam.edu.br, helder@ufam.edu.br,luizmiguel.ac2@gmail.com). **Escola Superior de Tecnologia, Universidade do Estado do Amazonas, AM (e-mail: mario_ruben14@hotmail.com)
\end{abstract}

\begin{abstract}
With the world becoming more technological, the use of computer programs for analysis and control activities has been growing more and more. For this work, it is shown how a man-machine interface using the programming language Python manages to show the behavior of the electrical quantities of an induction motor, in which this interface shows the data of these quantities in graphs in real time or in a given interval of time using devices well known in electronics, such as: Arduino and frequency inverter.
\end{abstract}

\begin{abstract}
Resumo: Com o mundo cada vez mais tecnológico, o uso de programas computacionais para atividades de análise e controle vem crescendo cada vez mais. Para este trabalho, é mostrado como uma interface homem-máquina utilizando a linguagem de programação Python consegue mostrar o comportamento das grandezas elétricas de um Motor de Indução, na qual essa interface mostra os dados dessas grandezas em gráficos em tempo real ou em um dado intervalo de tempo utilizando dispositivos muito conhecidos na eletrônica, tais como: Arduino e Inversor de Frequência.
\end{abstract}

Keywords: Man-machine interface; Python; Induction motor; Arduino; Frequency inverter; Electrical quatities; Graphs.

Palavras-chaves: Interface homem-máquina; Python; Motor de Indução; Arduino; Inversor de Frequência; Grandezas elétricas; Gráficos.

\section{INTRODUÇÃO}

Os sistemas de motores elétricos são a tecnologia que mais consomem energia na indústria (Trianni, 2019). Nos cursos de engenharia elétrica, mais especificamente na área de sistema elétricos de potência um conteúdo muito estudado por alunos em salas de aula e laboratórios é sobre maquinas elétricas. Nesse conteúdo estuda-se sobre o funcionamento de motores elétricos, um equipamento muito utilizado no dia a dia na vida das pessoas, como por exemplo: ventiladores, compressores, máquina de lavar, além de seu forte uso em indústrias e no comércio. Os motores de indução são os motores mais comuns utilizados em movimento. Eles são amplamente utilizados em aplicações industriais por sua simplicidade, design robusto, de baixo custo, baixa manutenção e conexão direta uma fonte de energia AC (Bieler \& Werneck, 2018). Em um estudo de Williamson ele concluiu que em países desenvolvidos países, pelo menos $60 \%$ do total de energia elétrica consumida pela indústria é responsável pela energia consumida pelos motores de indução (Bieler \& Werneck, 2018).

Os motores elétricos possuem uma série de grandezas elétricas durante seu funcionamento. Para medi-las, são necessários alguns instrumentos como: multímetros digitais ou analógicos, esses mostram ao usuário qual o valor numérico daquela grandeza naquele instante, e quando seu valor altera, o novo valor será mostrado no instrumento e o valor anterior é esquecido. Nesse caso, só é possível ver os resultados das grandezas naquele instante. Porém, existe uma forma na qual todos os valores que foram registrados no instrumento sejam guardados com o objetivo de plotar gráficos para estudos posteriores. Para isso, é necessário a construção de uma interface homem-máquina ou interface gráfica.

A interface homem-máquina é um meio entre humanos e computador para transmitir e trocar informações. É uma parte importante do sistema de computador, e é a mídia interação e troca de informações entre o sistema e o usuário. Ele será convertido para forma interna e a informação homemmáquina pode ser aceita por humanos (An \& Others, 2017). Essa interface gráfica é um meio que o usuário tem para conseguir ver todos os dados do motor através de gráficos utilizando softwares que trabalham com alguma linguagem de programação que pode ser como por exemplo: Python, C, Java. Através desse software é possível desenvolver um código com certa lógica de programação que adquiri todos os dados que do motor elétrico que chegam até esse código, e 
em seguida mostrá-los em gráficos, a linguagem que usada neste trabalho é o Python.

O Python é uma plataforma de código aberto, interpretada linguagem de programação que suporta tanto programação imperativa, programação funcional e orientada a objetos programação. Essa plataforma altamente popular em todo o mundo com sua elegância, compacidade e simplicidade, e tornou-se uma das mais populares programações de computadores línguas (Guanghui \& Others, 2018). O Python é uma linguagem de programação de alto nível que é amplamente utilizada por não-programadores e cientistas (Bingol \& Krishnamurthy, 2019).

Um dispositivo que também tem um papel importante no trabalho para que os dados do motor elétrico sejam levados para a interface no computador é o Arduino.

O Arduino é um microcontrolador de código aberto, que se tornou muito popular entre estudantes, entusiastas e profissionais devido ao seu baixo custo, a sua facilidade de uso e sua programação amigável tornando-o uma ótima ferramenta para teste de protótipos (Varanis, 2016). O Arduino é provavelmente o maior impacto do OSHW (Open Source Hardware) nos últimos anos. Sua abordagem, baseada simplicidade de uso, baixo custo e criação de comunidades, tem fez desta plataforma um grande sucesso. É usado por pessoas com habilidades muito diferentes, de iniciantes a especialistas. Tem sido adotado em diferentes tipos de organizações, desde a criação espaços ou escolas a universidades ou centros de pesquisa, e é usado com objetivos muito diferentes, desde a prototipagem até a pequena final produção em escala (Torroja \& Others, 2015). Através desse dispositivo é possível aquisitar as informações de grandezas do motor elétrico e são mostradas na interface. Mas que o Arduino e a interface consigam se comunicar, é importante citar o uso da comunicação serial.

Em geral, a comunicação entre dois dispositivos eletrônicos é realizada de modo serial, isto é, as informações são passadas bit a bit do transmissor para o receptor. Este tipo de comunicação possui algumas vantagens em relação à comunicação paralela, na qual a palavra de 8 bits (byte) é enviada toda de uma vez (Almeida, Moraes na Seraphim, 2016).

Neste trabalho é mostrado os conceitos de cada etapa para a construção dessa interface homem - máquina na seção 2 , a metodologia de funcionamento de cada etapa na seção 3 , e os resultados obtidos na prática na seção 4 , além de uma breve conclusão do trabalho com respeito a sua utilização em locais de estudo.

\section{INTERFACE HOMEM-MÁQUINA}

Conforme dito na introdução, o este artigo se baseia na construção de uma interface homem-máquina, na qual mostrarão dados das grandezas de um Motor de Indução elétrico através de gráficos. Para todo esse processo acontecer, outros elementos nesse sistema também estão inclusos como o Arduino, comunicação serial além de
Inversor de Frequência. Nas seções a seguir, são brevemente conceituados esses elementos.

\subsection{Motor de Indução elétrico}

O motor elétrico é um dispositivo que transforma energia elétrica em energia mecânica, em geral, energia cinética, ou seja, num motor, a simples presença da corrente elétrica, seja corrente contínua, ou alternada, garante dependendo da aplicação do motor (Franchi, 2008).

De acordo com o tipo de fonte de alimentação os motores elétricos podem ser divididos em motores de corrente contínua e de corrente alternada (Franchi, 2008).

O Motor de Indução converteu-se no tipo mais usado na indústria, porque a maioria dos sistemas atuais de distribuição de energia elétrica é de corrente alternada (Franchi, 2008).

Quanto a constituição do Motor de Indução (motor assíncrono), esse possui os elementos: circuito magnético estático, bobinas, rotor (Franchi, 2008).

O circuito magnético estático é composto de chapas ferromagnéticas empilhadas e isoladas entre si, ao qual se dá o nome estator, em que fica a carcaça que é a estrutura que também tem a função de suporte do conjunto. Possui uma construção robusta em ferro fundido, aço ou alumínio injetado, resistente à corrosão e com aletas para a refrigeração (Franchi, 2008).

Quanto as bobinas, de acordo com o número de grupos que caracterizam o motor monofásico ou polifásico; localizados em cavas abertas no estator e alimentados pela rede de corrente alternada (Franchi, 2008).

O rotor formado por um núcleo ferromagnético, também laminado, sobre o qual se encontra um enrolamento ou um conjunto de condutores paralelos, nos quais são induzidas corrente provocadas pela corrente alternada das bobinas do estator (Franchi, 2008).

\subsection{Interface gráfica}

As Interfaces Gráficas com Usuário (GUI, Graphic User Interface) se popularizaram no ambiente desktop, devido à facilidade de uso e a produtividade. Existem hoje muitas bibliotecas disponíveis para a construção de aplicações GUI, tais como: GTK+, QT, TK e wxWidgets (Borges, 2010).

Interfaces gráficas geralmente utilizam a metáfora do desktop, um espaço em duas dimensões, é que ocupado por janelas retangulares, que representam aplicativos, propriedades ou documentos (Borges, 2010).

As janelas podem conter diversos tipos de controles (objetos utilizados para interagir com o usuário ou para apresentar informações) e containers (objetos que servem de repositório para coleções de outros objetos) (Borges, 2010).

$\mathrm{Na}$ maior parte do tempo, a interface gráfica espera por eventos e responde de acordo. Os eventos podem ser 
resultado da interação do usuário, como cliques e arrastar de mouse ou digitação, ou ainda de eventos de sistema, como o relógio da máquina. A reação a um evento qualquer é definida através de funções callback (funções que são passadas como argumento para outras rotinas) (Borges, 2010).

\subsection{Linguagem Python}

O Python possui uma sintaxe clara e concisa, que favorece a legibilidade do código fonte, tornando a linguagem mais produtiva (Borges, 2010).

A linguagem inclui diversas estruturas de alto nível (listas, dicionários, data / hora, complexos e outras) e uma vasta coleção de módulos prontos para uso, além de frameworks de terceiros que podem ser adicionados (Borges, 2010.

Python utiliza tipagem dinâmica, o que significa que o tipo de uma variável é inferido pelo interpretador em tempo de execução (isto é conhecido como Duck Typing). No momento em que uma variável é criada através de atribuição, o interpretador define um tipo para a variável, com as operações que podem ser aplicadas (Borges, 2010).

A tipagem do Python é forte, ou seja, o interpretador verifica se as operações são válidas e não faz coerções automáticas entre tipos incompatíveis. Para realizar a operação entre tipos não compatíveis, é necessário converter explicitamente o tipo da variável ou variáveis antes da operação (Borges, 2010).

O código fonte é traduzido pelo Python para bytecode, que é um formato binário com instruções para o interpretador. $\mathrm{O}$ bytecode é multiplataforma e pode ser distribuído e executado sem fonte original (Borges, 2010).

Um programa feito em Python é constituído de linhas, que podem continuar nas linhas seguintes, pelo uso do caractere de barra invertida ( () ao final da linha ou parênteses, colchetes ou chaves, em expressões que utilizam tais caracteres (Borges, 2010).

Em Python, os blocos de código são delimitados pelo uso de indentação, que deve ser constante no bloco de código, porém é considerada uma boa prática manter a consistência no projeto todo e evitar a mistura de tabulações e espaços (Borges, 2010).

\subsection{Arduino}

O Arduino é um microcontrolador em que seus componentes se destinam ao fornecimento de energia elétrica e à comunicação com o seu computador. Ele tem um processador, 2 ou 2,5 quilobytes de memória de acesso aleatório (RAM) para guardar dados, 1 quilobyte de memória programável apagável somente de leitura (EPROM) e quilobytes de memória flash para armazenar os programas (Monk, 2014).

Outra característica do Arduino é a constituição de pinos de entrada e saída. Esses pinos estabelecem a conexão entre o microcontrolador e o resto da eletrônica (Monk, 2014).
As entradas podem ler dados tanto digitais quanto analógicos. Isso nos possibilita conectar muitos tipos diferentes de sensores de luz, temperatura, som e outros (Monk, 2014).

As saídas também podem ser analógicas ou digitais. Assim, é possível fazer que um pino esteja ativado ou desativado $(0$ volts ou 5 volts), permitindo que os diodos emissores de luz (LEDs) sejam ligados ou desligados diretamente, ou também se pode usar as saídas para controlar dispositivos com potências mais elevadas, como um motor. Esses pinos também podem fornecer uma tensão de saída analógica, ou seja, pode-se fazer a saída de um pino apresente uma dada tensão em particular (Monk, 2014).

O Arduino pode ser energizado por meio do conector USB que fica à direita, em cima, ou o soquete, $\mathrm{CC}$ que fica mais abaixo. Quando a alimentação é feita com um adaptador CC de tensão ou baterias, um valor entre $7,5 \mathrm{~V}$ e $12 \mathrm{~V}$ CC (Monk, 2014).

Quanto as entradas analógicas, elas são chamadas de "Analog In 0 to 5" (Entradas analógicas 1 a 5). Esses seis pinos podem ser usados para medir a tensão aplicada em cada um deles, permitindo que esses valores possam ser usados em um sketch. Essas entradas têm uma tensão e não uma corrente. Como essas entradas têm uma resistência interna muito elevada, a corrente que entra e vai para terra é muito pequena (Monk, 2014).

O monitor serial é uma ferramenta muito útil, especialmente para depuração de código. O monitor exibe os dados seriais enviados do Arduino (USB ou placa serial). Também pode-se enviar dados de volta ao Arduino utilizando o monitor serial (McRoberts, 2011).

A comunicação serial é o processo de envio de dados, um bit de cada vez, por um link de comunicação (McRoberts, 2011). É dessa forma que o Arduino consegue se comunicar com um computador e com um outro programa que nesse trabalho se trata da interface.

\subsection{Inversor de Frequência}

Uma grande infinidade de equipamentos foi desenvolvida para as mais diversas variedades de aplicações e setores industriais. Um dos equipamentos mais utilizados nesses processos juntamente com o CLP é o Inversor de Frequência. Um equipamento versátil e dinâmico que permitiu o uso de motores de indução para controle de velocidade em substituição aos motores de corrente contínua (Franchi, 2008).

O avanço da eletrônica de potência permitiu o desenvolvimento de conversores de frequência com dispositivos de estado sólido, inicialmente com tiristores e atualmente com transistores, mais especificamente o IGBT, transistor bipolar de porta isolada. Os cicloconversores antecederam, de certa forma, os atuais inversores. Eles eram utilizados para converter $60 \mathrm{~Hz}$ da rede em uma frequência mais baixa, era fim, em CA novamente (Franchi, 2008). 
Com esse avanço da tecnologia dos dispositivos semicondutores de potência, os inversores de frequência atuam de forma importante em toda indústria. O Inversor de Frequência convencional contém elementos armazenadores de energia no seu elo de corrente contínua. Estes elementos armazenadores de energia são capacitores eletrolíticos e possibilitam manter a tensão do ele de corrente contínua constante. Entretanto quanto maior sua capacitância maior a distorção da corrente de entrada. (Oliveira Filho, 2010)

Os inversores podem ser classificados pela sua topologia, que é dividida em três partes, sendo a primeira para o tipo de retificação de entrada, a segunda para o tipo de controle do circuito intermediário e a terceira para a saída (Franchi, 2008).

\section{METODOLOGIA}

O projeto interface homem-máquina é divido nas seguintes partes: Motor elétrico, Inversor de Frequência, Arduino, interface gráfica em Python. Cada um desses elementos exerce uma função.

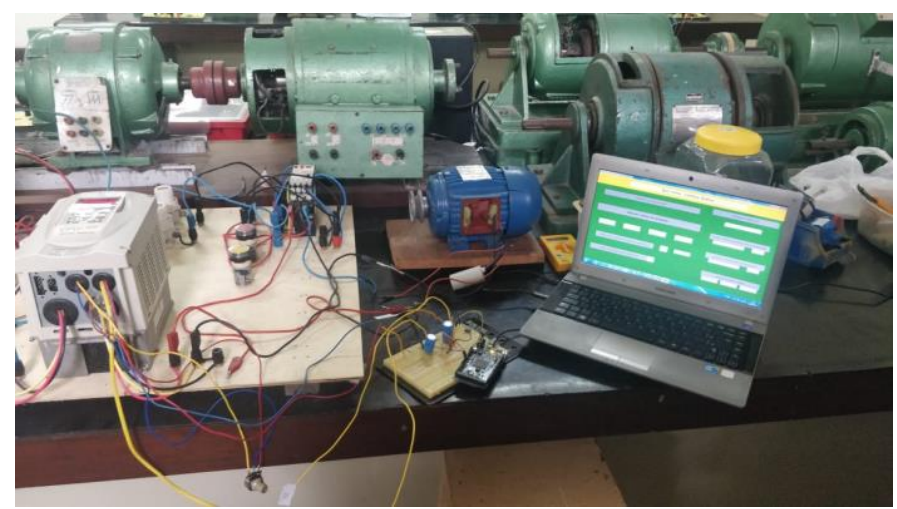

Fig. 1 Construção do sistema completo do motor para a interface

\subsection{Motor de Indução}

O motor elétrico de indução para a análise é bifásico, ou seja, alimentado em duas fases com $0,5 \mathrm{CV} / 370 \mathrm{~W}$ de potência. No mesmo serão analisadas grandezas elétricas: tensão, corrente e velocidade durante seu funcionamento.

Tabela 1. Dados e Parâmetros do Motor de Indução

\begin{tabular}{|c|c|}
\hline Parâmetros & Dados \\
\hline Modelo & 371 \\
\hline Potência & $0,5 \mathrm{CV} / 370 \mathrm{~W}$ \\
\hline Frequência & $60 \mathrm{~Hz}$ \\
\hline Tensão & $220 \mathrm{~V}$ \\
\hline Corrente & $2,7 \mathrm{~A}$ \\
\hline Rendimento & 68 \\
\hline Cosseno $\varphi$ & 68 \\
\hline Velocidade & $1700 \mathrm{RPM}$ \\
\hline Fator de serviço & 1,15 \\
\hline Classe de isolamento & $\mathrm{B}\left(120^{\circ} \mathrm{C}\right)$ \\
\hline
\end{tabular}

\begin{tabular}{|c|c|}
\hline Ip/In & 5 \\
\hline IP (Grau de proteção) & 55 \\
\hline$N^{\circ}$ de Fases & $1 \phi$ \\
\hline R.D. & 6203 \\
\hline R.T. & 6202 \\
\hline
\end{tabular}

\subsection{Inversor de Frequência CFW 09}

Para que essas grandezas sejam aquisitadas, é necessário um Inversor de Frequência CFW 09.

O Inversor de Frequência normalmente é usado para controle de partida de motores, porém, para o caso deste trabalho, será programado o inversor para que motor seja acionado com uma única velocidade em regime permanente, pois, o objetivo do trabalho é analisar com comportamento do motor.

As informações de tensão, corrente e velocidade do motor serão convertidas em valores de tensão dentro do próprio inversor de forma proporcional, pois, haverá um ganho na saída analógica que irá reduzir a tensão medida das três grandezas do motor, a qual representa de forma proporcional o valor real. O valor de saída do inversor de fábrica varia entre 0 a $10 \mathrm{~V}$.

Então, antes do inversor receber as informações do motor, primeiro é necessário programá-lo colocando os valores nominais do motor, porque será ele quem irá fornecer a energia ao motor para o mesmo funcionar.

\subsection{Arduino}

Após as grandezas tensão, corrente e velocidade serem convertidas em apenas valores na grandeza de tensão, eles podem ser levados ao Arduino, onde aquele sinal de tensão vindo do inversor será lido nas portas analógicas do Arduino e transformado em dados dentro do código do próprio Arduino. Por isso, é necessário transformar o sinal (dados) de cada grandeza em tensão, pois o Arduino lê apenas valores de tensão.

O Arduino usado foi o modelo DUE que tem sua alimentação em $3.3 \mathrm{~V}$.

Para o projeto, serão usadas três portas analógicas A1, A2, A3 onde ambas farão a leitura de tensão, corrente e velocidade respectivamente.

Os dados armazenados no Arduino serão passados para a interface através da comunicação serial entre esses dois programas.

\subsection{Interface homem-máquina}

E por fim, na interface homem-máquina ou interface gráfica, quando for solicitado, um gráfico tempo real ou por dado intervalo de tempo será plotado.

A seguir na figura 2 é mostrado o corpo de todo o trabalho. 


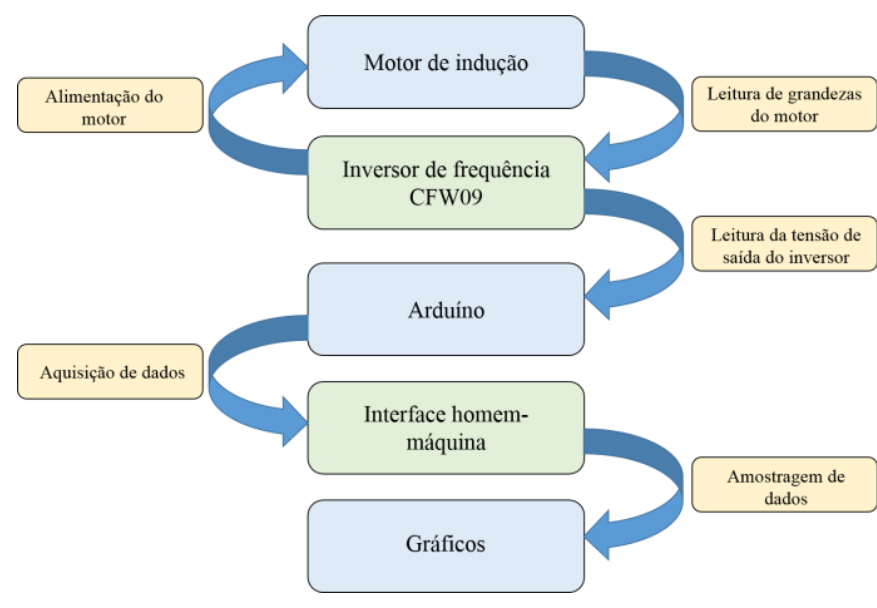

Fig. 2 Fluxograma do projeto

\section{RESULTADOS}

Com a interface homem-máquina, foi possível fazer os experimentos de aquisição de dados das grandezas do Mc de Indução.

\subsection{Inversor de Frequência-CFW 09}

Para realizar os experimentos, primeiramente foi necess: programar o Inversor de Frequência CFW 09. Durant programação do inversor, foram colocados os valc nominais do motor, valores de tensão, corrente, velocidac frequência da rede. Após colocar os valores nominal, ajustado o valor da velocidade em que o motor dev funcionar em regime permanente.

No inversor, foi necessário ajustar o intervalo de tensão que o CFW 09 iria enviar para o Arduino, visto que o mesmo só pode receber no máximo $3,3 \mathrm{~V}$ (Volts) de tensão nas suas entradas analógicas. Foi estimado um intervalo de 0 a $2,8 \mathrm{~V}$. Isso significa que se as amplitudes atingirem seus valores nominais, então a tensão de saída será a máxima que é 2,8 V.

\subsection{Interface Gráfica}

A interface utilizada no código em Python ficou da seguinte forma:

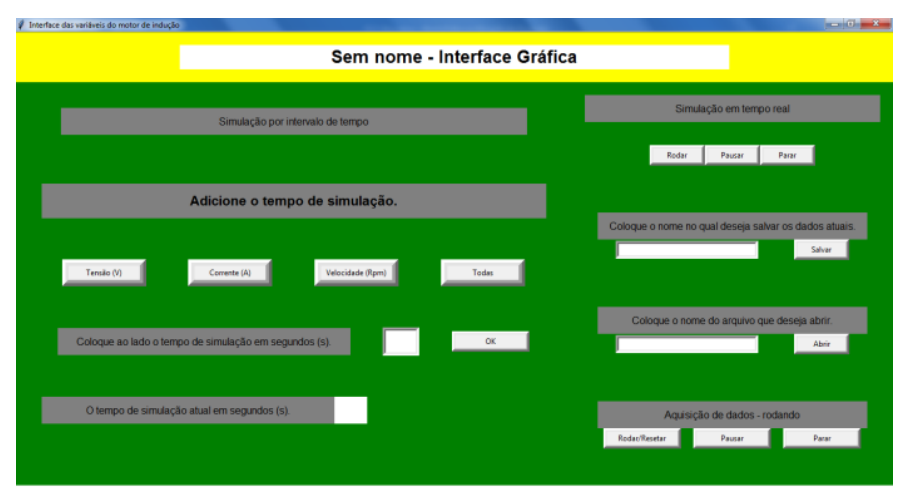

Fig. 3 - Interface Homem-Máquina
Nessa interface é possível plotar gráficos em tempo real, e também por um dado intervalo de tempo.

Para este artigo, optou-se por fazer a aquisição dos dados por intervalo de tempo durante 50 segundos.

É possível colocar o tempo que quiser na interface para plotar o gráfico através da caixa de texto. Além disso, também é possível salvar uma simulação, e posteriormente abri-la quando desejar.

\subsection{Gráficos}

Através das informações passadas do Arduino para o Python, foi possível plotar os gráficos que mostraram o comportamento do motor nas grandezas de tensão, corrente e velocidade.

Os gráficos a seguir mostram como o motor se comportou durante os 50 segundos.

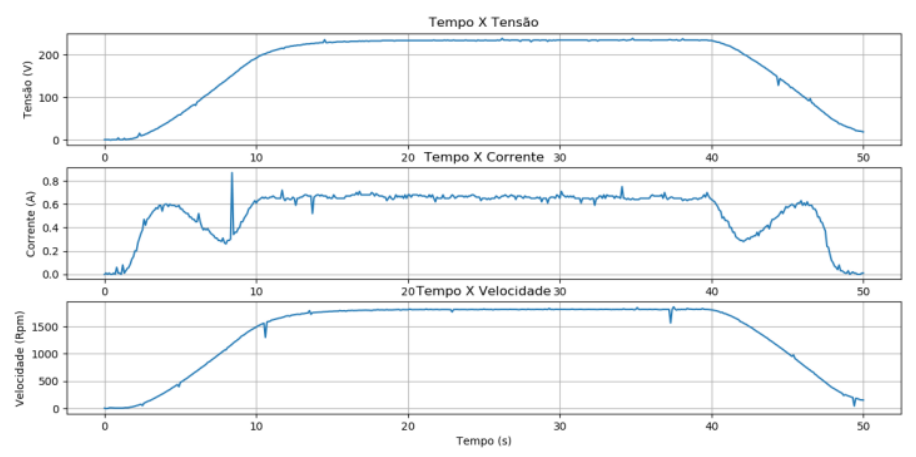

Fig. 4 Gráficos de tensão, corrente e velocidade

Durante o tempo simulado, o motor foi inicialmente acionado, em seguida permaneceu em movimento, e depois de um tempo ele foi desligado. Pode-se ver que isso foi nitidamente mostrado nos três gráficos.

No início dos três gráficos observa-se uma inclinação para cima, o que representa que o motor estava acelerando, ou seja, aumentando a velocidade, e por consequência a tensão e a corrente também se elevaram.

Quando o motor atingiu seu valor nominal, ou seja, velocidade máxima possível, ele ficou com seus valores de forma contínua.

E por fim, quando o motor foi desligado, criou-se uma inclinação para baixo, o que mostra que ele estava desacelerando, ou seja, diminuindo velocidade, e por consequência a tensão e a corrente também diminuíram.

O gráfico de tensão possui uma tensão variável, porque como a velocidade está variando, isso exige que a frequência da rede elétrica tenha que se alterar para que a velocidade do motor seja aquela em que está naquele momento. Isso faz com que a onda alternada da tensão da rede tenha uma frequência menor, ou seja, um período maior. Então, a tensão média dessa onda com período maior acaba sendo alterada 
proporcionalmente a frequência. $\mathrm{O}$ gráfico de tensão representa a tensão média no motor.

Agora um detalhe que se observou em todos os gráficos foi que todos tiveram oscilações, ou seja, ruídos. Esses ruídos acontecem porque o Arduino ao ler o valor de tensão do CFW 09, ele ler com certa imprecisão fazendo com que apareça no gráfico valores irreais a respeito do motor.

\section{CONCLUSÕES}

O uso da interface gráfica para o estudo do motor elétrico pode impactar tanto na área de pesquisas quanto na área comercial. Através desse projeto, é possível que o mesmo possa virar um produto que muitas indústrias utilizariam para analisar se seus motores estão partindo corretamente, e se seu desenvolvimento em regime permanente está atuando de forma correta. Além disso, em escolas e universidade, isso pode se tornar uma ferramenta de estudo para os alunos que precisarem ver o comportamento do motor na prática, o que facilita a compreensão.

As principais vantagens desse projeto são que a linguagem Python usada no projeto é muita conhecida, fácil uso e aprendizagem. Além disso, o projeto se dispõe de circuitos muito conhecidos na área da eletricidade em sua construção, tais como o Arduino e um Filtro, e ambos são de pequeno porte e fácil de transportá-los. Todos os dispositivos citados são de baixo custo e facilmente são encontrados no mercado. Porém, o Inversor de Frequência, que possui dimensões de tamanho médio e um custo mais elevado, pode facilmente ser substituído por equipamentos mais simples como um TP (Transformador de potencial) e um TC (Transformador de corrente), os quais fariam a leitura das grandezas do motor no lugar do Inversor de Frequência.

De modo geral, o trabalho descrito neste artigo, demostra ter uma boa aplicação no mercado e nas universidades com um baixo custo e fácil compreensão. Visto que o mesmo ficaria em constante aprimoramento.

\section{AGRADECIMENTOS}

Este trabalho teve o apoio financeiro do projeto QUALIPIM-CAP; Capacitação de Recursos Humanos para o Polo Industrial de Manaus em automação e otimização de processos e produção industriais, no âmbito do Projeto Prioritário de Formação de Recursos Humanos SUFRAMA/CAPDA, Fundação Muraki.

\section{REFERÊNCIAS}

Almeida, R. M. A., Moraes, C. H. V. \& Seraphim, T. F. P. (2016). CAPÍTULO 18 - Comunicação serial. Rodrigo Maximiano Antunes de Almeida, Carlos Henrique Valério de Moraes \& Thatyana de Faria Piola Seraphim, Programação de Sistemas Embarcados, 271-305, Elsevier Editora Ltda.

An, F. \& Others (2017). The Amelioration of Vertical Elevator Man-Machine Interface Based on the Multi Channel Theory of Ergonomics. Hangzhou, 81-84.
Bieler, G. \& Werneck, M. M. (2018). A magnetostrictivefiber Bragg grating sensor for induction motor health monitoring. Measurement, volume (122), 117-127.

Bingol, O. R. \& Krishnamurthy, A. (2019). NURBS-Python: An open-source object-oriented NURBS modeling framework in Python. SoftwareX, volume (9), 85-94.

Borges, L. E. (2010). Python Para Desenvolvedores, 13-23, Edição do autor, Rio de Janeiro - RJ.

Franchi, C. M. (2008). ACIONAMENTOS ELÉTRICOS, $17-$ 22, Editora Érica Ltda., São Paulo - SP.

Guanghui, Z. \& Others (2018). Case-Based Teaching Organization for Python Programming that Focuses on Skill Training. Colombo, 1-5.

McRoberts, M. (2011). ARDUINO BÁSICO, 34;89 ,Novatec Editora Ltda., São Paulo - SP.

Monk, S. (2014). 30 PROJETOS COM ARDUINO, 20-23, Bookman editora Ltda., Porto Alegre - RS.

Oliveira Filho, M \& Others (2010). Um controlador de corrente de carga para o conversor em matriz trifásico para trifásico e para o Inversor de Frequência sem capacitor do elo de corrente contínua. Sba Controle \& Automação, Campinas - SP.

Torroja, Y. \& Others (2015). A serial port based debugging tool to improve learning with Arduino. Esroril, 1-4.

Trianni, A., Cagno, E. \& Accordini, D. (2019). A review of Energy Efficiency Measures Within Electric Motors Systems. Energy Procedia, volume (158), 3346-3351.

Varanis, Marcus \& Others (2016). Instrumentation for mechanical vibrations analysis in the time domain and frequency domain using the Arduino Platform. Rev. Bras. Ensino Fís, São Paulo - SP. 